\title{
Umwandlung von Chlor 35 mit thermischen Neutronen unter Aussendung von Protonen
}

\author{
Von Werner Maurer* \\ (Z. Naturforschg. 4 a, 150-152 [1949]; eingegangen am 6. September 1948)
}

\begin{abstract}
Mit Chlor von geänderter Isotopenzusammensetzung wird gezeigt, daß das langlebige Schwefelisotop von 88 Tagen $\mathrm{HZ}$ die Massenzahl 35 hat und bei der Neutronenbestrahlung von Chlor durch die Reaktion ${ }_{17} \mathrm{Cl}^{35}(\mathrm{n}, \mathrm{p}){ }_{16} \mathrm{~S}^{35}$ (88 Tage) entsteht.

Diese Umwandlung verläuft auch mit thermischen Neutronen. Aus Aktivitätsmessungen ergab sich ein Wirkungsquerschnitt von $0,29 \cdot 10^{-24} \mathrm{~cm}^{2}$, bezogen auf reines $\mathrm{Cl}^{35}$. Der Wirkungsquerschnitt des Prozesses ${ }_{30} \mathrm{Zn}^{64}$ (n, p) ${ }_{29} \mathrm{Cu}^{64}$ (12,8 Stdn.), der energetisch auch mit thermischen Neutronen verlaufen müßte, ergab sich $\mathrm{zu}<10^{-29} \mathrm{~cm}^{2}$. Diese Wirkungsquerschnittsmessungen stehen im Einklang mit einfachen theoretischen Cherlegungen.

Es werden Angaben über die Ausbeute an $\mathrm{S}^{25}$-Präparaten gemacht.
\end{abstract}

$\mathrm{B}$ ei der Bestrahlung von Chlor mit schnellen Neutronen entsteht ein aktives Schwefelisotop mit einer Halbwertszeit (HZ) von 88 Tagen. Da die obere Grenze seines $\beta$-Spektrums nur $107 \mathrm{kV}$ beträgt, muß die Kernreaktion energetisch auch mit thermischen Neutronen verlaufen können. Die Protonenenergie sollte dann gleich der Differenz von Neutronen- und Protonenmasse vermindert um $107 \mathrm{kV}$ sein. Das ergibt einen Betrag von $650 \mathrm{kV}$.

Der Wirkungsquerschnitt (WQ) dieser Reaktion ist von $\mathrm{K}$ a me $\mathrm{n}^{\mathbf{1}}$ für ein Neutronenspektrum von maximal $20 \mathrm{eMV}$ zu $2 \pm 1 \cdot 10^{-27} \mathrm{~cm}^{2}$ gemessen worden. Nach der gleichen Arbeit ist der Effekt mit Paraffin verstärkbar und mit Cadmium absorbierbar. Er verläuft also auch mit thermischen Neutronen.

Bisher sind fünf Umwandlungen mit thermischen Neutronen unter Aussendung von geladenen Teilchen bekannt. Es sind dies $\operatorname{Li}^{6}(n, \alpha), B^{10}(n, \alpha)$, $\mathrm{B}^{10}(n, p), \mathrm{N}^{14}(n, p)$ und $\mathrm{F}^{19}(\mathrm{n}, \alpha)$. Eine weitere Umwandlung dieser Art bei Chlor mit seiner hohen Ordnungszahl 17 ist erstens prinzipiell und zweitens im Hinblick auf eine Möglichkeit zur Herstellung starker Schwefelpräparate von Interesse. Im folgenden wird über eine Wiederholung des Kamenschen Versuchs unter verbesserten Bedingungen berichtet, über die Messung des WQ von $\mathrm{Cl}^{35}(\mathrm{n}, \mathrm{p}) \mathrm{S}^{35}$ für thermische Neutronen und über die Zuordnung des Prozesses mit an $\mathrm{Cl}^{37}$ angereichertem Chlor.

* Köln-Lindenthal, Lindenburg Haus 3 b.
Das langlebige Schwefel-Isotop ist herstellbar durch die Prozesse $\mathrm{Cl}(\mathrm{n}, \mathrm{p})$ und $\mathrm{S}(\mathrm{d}, \mathrm{p})$. Danach kommen die Massenzahlen 35 oder 37 in Frage. Unter der Annahme, daß die Masse = 35 ist, fand $\mathrm{K}$ a $\mathrm{m}$ e $\mathrm{n}^{1}$ für den dann vorliegenden $\mathrm{S}^{34}(\mathrm{~d}, \mathrm{p}) \mathrm{S}^{35}$ Prozeß bei einer Deuteronenenergie von 5 bis $14 \mathrm{eMV}$ einen WQ von $7 \pm 3 \cdot 10^{-27} \mathrm{~cm}^{2}$. Wenn der $\mathrm{S}(\mathrm{d}, \mathrm{p})$-Prozeß von dem im Vergleich mit $\mathrm{S}^{34}$ 270-mal selteneren $S^{36}$ ausgehen sollte, würde sich ein unwahrscheinlich hoher WQ ergeben. Kamen ordnete deshalb den langlebigen Schwefel der Masse $35 \mathrm{zu}$.

Eine ganz sichere Deutung ist möglich, wenn man Chlor von geänderter Isotopenzusammensetzung bestrahlt. Ein solcher Versuch wurde durchgeführt. Das gewöhnliche Chlor besteht aus $75 \% \mathrm{Cl}^{35}$ und $25 \% \mathrm{Cl}^{37}$. Es standen $460 \mathrm{mg} \mathrm{NaCl}$ mit $48 \% \mathrm{Cl}^{35}$ und $52 \% \mathrm{Cl}^{37}$ zur Verfügung ${ }^{2}$. Dieses Präparat wurde gleichzeitig mit $460 \mathrm{mg}$ gewöhnlichem $\mathrm{NaCl}$ mit Neutronen bestrahlt. Die Neutronenintensität an der Stelle der beiden NaClPräparate war gleich. Nach der Bestrahlung wurde der gebildete aktive Schwefel nach Zugabe von inaktiver $\mathrm{H}_{2} \mathrm{SO}_{4}$ als $\mathrm{BaSO}_{4}$ gefällt. Wenn der Schwefel aus $\mathrm{Cl}^{35}$ entsteht, sollte gewöhnliches $\mathrm{NaCl}$ einen 1,58-mal stärkeren Effekt liefern als das oben beschriebene, an $\mathrm{Cl}^{35}$ abgereicherte $\mathrm{NaCl}$. Bei $\mathrm{Cl}^{37}$ als Ausgangsisotop würde das Verhältnis 0,48 betragen. Der Versuch lieferte das Verhältnis

1 M. D. K a m e n, Physic. Rev. 60, 537 [1941].

${ }^{2}$ Hrn. Prof. Clu si us möchte ich auch an dieser Stelle herzlich für die Herstellung und bereitwillige Úberlassung des Chlor-Präparats danken. 
1,48 $\pm 0,1$. Das langlebige Schwefel-Isotop entsteht also sicher aus $\mathrm{Cl}^{35}$ durch den Prozeß

$$
\mathrm{Cl}^{35}(\mathrm{n}, \mathrm{p}) \mathrm{S}^{35} \text { (88 Tage), }
$$

bzw. bei der Deuteronenbestrahlung von Schwefel durch den Prozeß

$$
\mathrm{S}^{34}(\mathrm{~d}, \mathrm{p}) \mathrm{S}^{35} \text { (88 Tage). }
$$

Die Zuordnung ist damit endgültig sichergestellt.

Um zu zeigen, daß die Umwandlung von Chlor mit thermischen Neutronen möglich ist, wurde folgender Versuch durchgeführt:

Zwei kleine, gleichgroße, mit je $3 \mathrm{~g} \mathrm{NH}_{4} \mathrm{Cl}$ gefüllte Behälter, von denen der eine mit Cadmium umgeben war, befanden sich im Innern eines Paraffinblocks. Ihre gegenseitige Entfernung betrug $7 \mathrm{~cm}$. Das Ganze wurde mit Neutronen von $\mathrm{Be}+\mathrm{d}$ bestrahlt, derart, daß sich beide Kästchen in äquivalenter Stellung zur Neutronenquelle und zum Paraffin befanden. Nach der Bestrahlung wurde das gebildete $\mathrm{S}^{35}$ aus' dem $\mathrm{NH}_{4} \mathrm{Cl}$ als $\mathrm{BaSO}_{4}$ abgetrennt. Die $\mathrm{S}^{35}$-Präparate waren frei von $\mathrm{P}^{32}$, der gleichzeitig durch die Reaktion $\mathrm{Cl}^{35}(\mathrm{n}, \boldsymbol{\alpha})$ $\mathrm{P}^{32}$ entsteht. Zur Messung der sehr weichen $\beta$-Strahlen des $\mathrm{S}^{35}$ von maximal $107 \mathrm{kV}$ diente ein $5 \mu-\mathrm{Al}-\mathrm{Zähler}$. Dieser war in ein Glasgefäß mit entsprechenden Schliffen zum Einbringen der $\mathrm{S}^{35}$-Präparate eingebaut.

Der Versuch ergab, daß die $\mathrm{S}^{\mathbf{3 5}}$-Präparate aus dem mit Cadmium abgeschirmten $\mathrm{NH}_{4} \mathrm{Cl}$ etwa 12-mal schwächer waren als die aus dem unabgeschirmten $\mathrm{NH}_{4} \mathrm{Cl}$. $\mathrm{Ka}$ men ${ }^{1}$ fand, bei sicher anderer Geometrie, den Faktor 6. Die Bildung von $\mathrm{S}^{35}$ erfolgte in der hier gewählten Anordnung also fast ausschließlich durch thermische Neutronen. Wenn zur Bestrahlung nicht die Neutronen von $\mathrm{Be}+\mathrm{d}$, sondern die von $\mathrm{Li}+\mathrm{d}$ genommen wurden, betrug das Intensitätsverhältnis 8. Dieser - verglichen mit oben - kleinere Wert erklärt sich durch die bei Lithium-Neutronen sehr wahrscheinlich relativ stärkere Erzeugung von aktivem Schwefel durch schnelle Neutronen. Diese liefern zu beiden Präparaten einen gleichen zusätzlichen Anteil.

Der WQ der Reaktion $\mathrm{Cl}^{35}(\mathrm{n}, \mathrm{p})$ für thermische Neutronen wurde durch Anschluß an den bekannten Wert für $\operatorname{Mn}(n, \gamma)$ gemessen. Wie oben wurden zwei gleiche Mengen $\mathrm{NH}_{4} \mathrm{Cl}$ in identischen Behältern bestrahlt, wobei der eine mit Cadmium umgeben war. Beiden $\mathrm{NH}_{4} \mathrm{Cl}$-Proben waren $100 \mathrm{mg}$ $\mathrm{MnCO}_{3}$ sorgfältig beigemischt worden. Dann wurde, wie oben bereits beschrieben, mit Neutronen bestrahlt. Aus der relativen Aktivität von $\mathrm{S}^{35}$ und $\mathrm{Mn}^{56}$ ließ sich dann der gesuchte WQ berechnen. Unter der Annahme eines Absorptionsquerschnitts von $11,2 \cdot 10^{-24} \mathrm{~cm}^{2}$ für $\operatorname{Mn}(\mathrm{n}, \gamma)$ ergab sich für die Umwandlung von reinem $\mathrm{Cl}^{35}$,mit thermischen Neutronen der überraschend große Wert von $0,29 \cdot 10^{-24} \mathrm{~cm}^{2} \pm 25 \%$. Für das natürliche Isotopengemisch ergibt sich ein WQ von $0,22 \cdot 10^{-24} \mathrm{~cm}^{2}$. Die Absorption der sehr weichen $\beta$-Strahlung von $\mathrm{S}^{35}$ in der $5 \mu-\mathrm{Al}$-Folie des Zählers und in der $\mathrm{BaSO}_{4}$-Schicht des Präparates wurde durch Versuche ermittelt und berücksichtigt.

Gibert, Roggen und Rossel ${ }^{3}$ haben die Energieverteilung der Protonen der Umwandlung $\mathrm{Cl}^{35}$ (n, p) mit Ionisationskammer und Proportionalverstärker gemessen. Aus der Protonen-Häufigkeit im Viergleich zu dem Prozeß $\mathrm{N}^{14}(n, p)$ wurde der $W Q$ von $\mathrm{Cl}^{35}(n, p)$ erschlossen. Für den $W Q$ für thermische Neutronen ergab sich ein 5-mal kleinerer Wert als für $\mathrm{N}^{14}(n, p)$. Wenn man für letzteren den Wert $1,2 \cdot 10^{-24} \mathrm{~cm}^{2}$ annimmt, so ergibt sich danach für $\mathrm{Cl}^{35}(\mathrm{n}, \mathrm{p})$ der $\mathrm{WQ}=0,24 \cdot 10^{-24} \mathrm{~cm}^{2}$, in guter Ubereinstimmung mit dem hier aus Aktivitätsmessungen ermittelten Wert. Es sei darauf hingewiesen, daß bei der Auszählung der Protonen auch ein evtl. vorhandener Prozeß $\mathrm{Cl}^{37}(n, p)$ mitgezählt würde, während bei der Aktivitätsmessung nur der Prozeß $\mathrm{Cl}^{35}(n, p)$ erfaßt wird. Offenbar spielt die Kernreaktion $\mathrm{Cl}^{37}(n, p)$ nur eine untergeordnete Rolle. Bei den hier beschriebenen Versuchen wurde die Schwefel-Aktivität über 6 Monate verfolgt. Sie fiel mit der bekannten $\mathrm{HZ}$ von 3 Monaten ab.

Für den Gesamtabsorptionsquerschnitt des gewöhnlichen Chlors für thermische Neutronen fanden Lapointe und Rasetti ${ }^{4} 27 \cdot 10^{-24} \mathrm{~cm}^{2}$, $\mathrm{Vol} \mathrm{z}^{5} 24 \cdot 10^{-24} \mathrm{~cm}^{2}$ und $\mathrm{R}$ a m m $32 \cdot 10^{-24} \mathrm{~cm}^{2}$. Danach sollte er mit hier genügender Genauigkeit etwa $30 \cdot 10^{-24} \mathrm{~cm}^{2}$ betragen. Er setzt sich nach bisheriger Kenntnis zusammen aus drei Teilprozessen: $\mathrm{Cl}^{35}(\mathrm{n}, \gamma), \mathrm{Cl}^{37}(\mathrm{n}, \gamma)$ und dem hier beschriebenen Prozeß $\mathrm{Cl}^{35}(\mathrm{n}, \mathrm{p})$. Für den Anlagerungsquerschnitt $\mathrm{Cl}^{37}(\mathrm{n}, \gamma)$ fanden $\mathrm{O}^{\prime} \mathrm{Neal}$ und Goldhaber ${ }^{6} \quad 0,07 \cdot 10^{-24} \mathrm{~cm}^{2}$ und Simna und

3 A. Gibert, I. R o g g e n u. I. R os s e l, Helv. physica Acta 17, 97 [1944].

${ }_{4}$ C. L a p o inte u. F. R a setti, Physic. Rev. 58, 869 [1940].

5 H. Volz, Z. Physik 121, 201 [1943].

6 D. D. O'Neal u. M. Goldhaber, Physic. Rev. 59, $102 \cdot[1941]$. 
Yamasaki ${ }^{\top} \quad 0,10 \cdot 10^{-24} \mathrm{~cm}^{2}$. Alle Zahlen beziehen sich auf das natürliche Isotopengemisch von Chlor. Der Teilquerschnitt von $\mathrm{Cl}^{35}(\mathrm{n}, \gamma)$ sollte danach $30-(0,22+0,10) \cdot 10^{-24} \mathrm{~cm}^{2}$, d. h. rund $30 \cdot 10^{-24} \mathrm{~cm}^{2}$ betragen. Eine direkte Messung wie bei $\mathrm{Cl}^{37}(\mathrm{n}, \gamma)$ liegt nicht vor, da über ein aktives Chlor der Masse 36 nichts bekannt ist.

Der bei der Anlagerung eines thermischen Neutrons an $\mathrm{Cl}^{35}$ gebildete, angeregte $\mathrm{Cl}^{36}$-Kern kann also in zwei Richtungen zerfallen: einmal durch Emission eines $\gamma$-Quants mit einem WQ von $\mathrm{rd}$. $30 \cdot 10^{-24} \mathrm{~cm}^{2}$, oder durch Emission eines Protons mit einem WQ von $0,29 \cdot 10^{-24} \mathrm{~cm}^{2}$. Die Emission eines $\gamma$-Quants ist also rd. 100-mal wahrscheinlicher als die eines Protons. Für dieses Proton beträgt die Durchdringungswahrscheinlichkeit durch den Potentialberg von Chlor nach Gamov etwa $10^{-4}$. Da bei der Maximalenergie die Laufzeit des Protons durch den Cl-Kern etwa $10^{-20} \mathrm{sec}$ beträgt, sollte die $\mathrm{HZ}$ für alleinigen Protonenzerfall, in Abwesenheit eines konkurrierenden Prozesses, etwa $10^{-16}$ sec betragen. Dabei ist noch nicht berücksichtigt, daß ein einzelnes Proton nur während eines kleinen Zeitbruchteils die gesamte Anregungsenergie des $\mathrm{Cl}^{36}$ auf sich vereinigt. Die HZ für Protonenzerfall ist also sicher wesentlich größer. Nun muß bei alleinigem $\gamma$-Zerfall des angeregten $\mathrm{Cl}^{36}$ eine $\mathrm{HZ}$ von etwa $10^{-16} \mathrm{sec}$ angenommen werden. Da nach obigem die Protonenemission 100-mal seltener ist als die $\gamma$-Emission, so wäre bei alleinigem Protonenzerfall eine $\mathrm{HZ}$ von etwa $10-{ }^{14} \mathrm{sec}$ zu erwarten. Dieser Wert ist rd. 100-mal größer als der, der sich aus dem Gamov-Faktor für ein Proton maximaler Energie ergibt. Für die Wahrscheinlichkeit dafür, daß ein einzelnes Proton die gesamte Anregungsenergie auf sich vereinigt, ergäbe sich damit ein Wert von $10^{-2}$, was durchaus verständlich erscheint. Von Weißkopf und Ewing sind diese Verhältnisse näher durchgerechnet worden. Ihre Ergebnisse sind aber auf einen so leichten Kern wie Cl nicht mehr anwendbar.

Immer dann, wenn die maximale $\beta$-Energie eines Elektronenstrahlers kleiner ist als die Differenz der Neutronen-Protonenmasse, sollte er durch einen $(n, p)$-Prozeß mit thermischen Neutronen entstehen können. Der einzige weitere bekannte Fall dieser Art ist der Prozeß $\mathrm{Zn}^{64}(\mathrm{n}, \mathrm{p}) \mathrm{Cu}^{64}$ (12,8 Stdn.). Da die maximale $\beta$-Energie von $\mathrm{Cu}^{64}=580 \mathrm{kV}$ ist, sollten die Protonen eine Energie von: Neutronenmasse - Protonenmasse $-580 \mathrm{kV}$ $=180 \mathrm{kV}$ haben. Für die Durchdringungswahrscheinlichkeit dieser Protonen durch den Potentialberg von $Z^{65}$ ergibt sich ein Wert von rd. 10-16, für alleinigen Protonenzerfall des angeregten $\mathrm{Zn}^{65}$ also eine $\mathrm{HZ}$ von rd. $10^{-5}$ sec. Für die Laufzeit des Protons im Kern wurde dabei ein Wert von 10-21 sec benutzt. Die Protonenemission sollte also $10^{11}$-mal seltener sein als die $\gamma$-Emission. Mit einem WQ des Zn für thermische Neutronen von maximal $10^{-24} \mathrm{~cm}^{2}$ ist für $\mathrm{Zn}(\mathrm{n}, \mathrm{p})$ ein $\mathrm{WQ}$ von maximal $10^{-35} \mathrm{~cm}^{2} \mathrm{zu}$ erwarten. Ein Versuch ergab als obere Grenze einen Wert von $10^{-29} \mathrm{~cm}^{2}$, was obiger Überlegung entspricht.

Um einen Begriff von den erreichbaren Präparatstärken von $\mathrm{S}^{35} \mathrm{zu}$ geben, sei angegeben, daß man bei Bestrahlung von $1 \mathrm{~kg}$ Ammoniumchlorid mit thermischen Neutronen von $1 \mathrm{~kg}(\mathrm{Ra}+\mathrm{Be})$ Aquivalent in 1 Stde. etwa $1 \mu$ Curie erhält.

7 K. Simna u. F. Y a masaki, Physic. Rev. 59, 402 [1941].

8 S. Flüg g e u. J. M a t t a u ch, Kernphysikalische Tabellen, Springer-Verlag, 1942. 